\title{
Germanica
}

\section{Franz Werfel entre expressionnisme et «Neue Sachlichkeit»}

Franz Werfel zwischen expressionismus und Neue Sachlichkeit

\section{Michel Reffet}

\section{(2) OpenEdition}

\section{Journals}

Édition électronique

URL : http://journals.openedition.org/germanica/2392

DOI : 10.4000/germanica.2392

ISSN : 2107-0784

\section{Éditeur}

Université de Lille

\section{Édition imprimée}

Date de publication : 31 décembre 1991

Pagination : 191-215

ISSN : 0984-2632

\section{Référence électronique}

Michel Reffet, «Franz Werfel entre expressionnisme et «Neue Sachlichkeit» », Germanica [En ligne], 9 |

1991, mis en ligne le 17 juillet 2014, consulté le 06 octobre 2020. URL : http://

journals.openedition.org/germanica/2392 ; DOI : https://doi.org/10.4000/germanica.2392

Ce document a été généré automatiquement le 6 octobre 2020.

(c) Tous droits réservés 


\title{
Franz Werfel entre expressionnisme et «Neue Sachlichkeit»
}

\author{
Franz Werfel zwischen expressionismus und Neue Sachlichkeit
}

\author{
Michel Reffet
}

1 Dans le panorama de l'histoire littéraire, Werfel reste d'une part le poète réunissant tous les thèmes et les traits caractéristiques de l'expressionnisme ${ }^{1}$, d'autre part le romancier croyant et militant du Chant de Bernadette. Il proclamait en 1915: «Dieu mesure seulement le degré de ferveur de nos battements de cœur $»^{2}$. En 1917 «Sachlichkeit» était pour lui « un mot dont se gargarisent aujourd'hui tous ceux qui s'abusent eux-mêmes et qui mentent au monde entier $»^{3}$. Il persistait en 1941 :

Déjà au temps où j'écrivais mes premiers vers, je m'étais juré, toujours et partout dans mes écrits, de magnifier le secret divin et la sainteté humaine - sans souci de notre époque qui se détourne par la raillerie, la haine et l'indifférence de ces dernières valeurs de notre vie ${ }^{4}$.

2 Faire la place d'un Werfel dans un courant qu'on a rendu en français par «l'ordre froid $»^{5}$, n'est-ce donc pas solliciter abusivement son message, vouloir le «caser » de force pour marquer son centenaire? Ce serait oublier qu'entre la décennie expressionniste 1910-1920 et sa phase d'authenticité religieuse 1930-1945, ni sa production, ni sa notoriété n'ont connu de relâchement. En 1930, Herbert Ihering, qui certes n'était pas ami de Werfel, continuait à écrire que celui-ci était le plus célèbre des écrivains de langue allemande ${ }^{6}$.

3 En 1920, l'expressionniste Werfel aborde la nouvelle décennie dans un effort d'objectivité qui le fait passer du lyrisme au théâtre. Critique sociale, pacifisme et idéalisme étaient assez minces dans le lyrisme. Celui-ci «n'était révolutionnaire que par rapport aux exigences du patriotisme $»^{7}$. «Finalement, il fallut, au lieu du poème, le drame pour servir la renaissante volonté d'agir $»^{8}$. Dès avant 1920 , certes, Werfel chargeait ses premières ébauches dramatiques d'un message politique. Le dialogue philosophique Euripides oder Über den Krieg et Die Troerinnen, adaptation d'Euripide, sont des œuvres pacifistes. Le fragment Stockleinen (1917) soulève déjà le problème de la Révolution tournant en dictature ${ }^{9}$. Malheureusement ces débuts sont obérés par de 
grandes faiblesses dramaturgiques. On assiste plutôt, comme le conclue une étude sur Die Troerinnen, à «l'hésitation du drame entre la Révolution historique et la transfiguration métaphysique $»^{10}$. Ces flottements disparaissent de la production des Années Vingt. Mais avant la mise en place d'une véritable structure politique dans son théâtre, Werfel aura dû faire lui-même l'expérience de la Révolution.

\section{Le théâtre des années vingt}

4 «Le premier homme, écrit Emil Staiger, qui a sauté sur une pierre, sur une élévation, pour haranguer quelques personnes, leur montrer qu'il voyait plus loin qu'elles, celuilà a déjà préparé la scène ${ }^{11}$. Cette phrase rend parfaitement compte de l'évolution de Werfel. Se débattant avec la technique dramatique, mécontent de ses premières pièces, il n'a trouvé le débouché qu'après une très courte, mais très intense période d'activisme. Au cours des journées révolutionnaires de novembre 1918 à Vienne, lui aussi grimpa sur une borne pour s'adresser à la foule et la lancer contre les banques ${ }^{12}$. La légalité ayant triomphé, il fut convoqué au commissariat de police pour s'expliquer. Il avoua tout, mais ajouta être adhérent du christianisme primitif et comme tel opposé à toute violence; son discours n'aurait eu pour but que de retenir les masses surexcitées de se livrer à des excès ${ }^{13}$. Mauvaise foi ? Que non pas. Le pacifiste était aussi un non violent, et cela va éclairer son théâtre des Années Vingt.

5 De 1920 à 1930, il donne six grands drames : Spiegelmensch (1920), Bocksgesang (1921), Schweiger (1922), Juarez undMaximilien (1924), Paulus unter den Juden (1926) et Das Reich Gottes in Böhmen (1930). L'interprétation de ce théâtre est restée longtemps problématique, car on l'abordait avec les critères psychodramatiques traditionnels. On a essayé leur appliquer la structure du conflit père-fils, thème majeur du théâtre expressionniste, et qui affleure aussi dans les trois premières pièces à examiner maintenant. Mais cela ne fonctionnait pas non plus, le rapport père-fils restant très accessoire et disparaissant complètement des trois pièces historiques. Si bien qu'on a renoncé à y trouver une cohérence ${ }^{14}$.

6 Or la structure de ce théâtre est politique. De ce fait déjà il s'inscrit dans la Neue Sachlichkeit. Si on les aborde sous l'angle politique, les drames des Années Vingt sont d'une parfaite cohérence. Toute autre approche les rend hétérogènes. Ils comportent deux différences essentielles par rapport aux débuts dramatiques: d'abord Werfel abandonne le style lyrique ou philosophique, d'autre part, la Révolution devient un véritable thème dramatique.

7 Les six pièces envisagées présentent tout d'abord des structures d'oppression politique tout-à-fait pertinente d'une pièce à l'autre. Ce sont des monstres (Spiegelmensch), les occupants turcs dans les Balkans (Bocksgesang), le fascisme en la personne d'un psychanalyste réactionnaire (Schweiger). Dans les trois drames historiques règnent les empereurs : dans Juarez und Maximilian ce sont François-Joseph et Napoléon III ; les crimes de Napoléon $\mathrm{I}^{\mathrm{er}}$ en Espagne sont évoqués ${ }^{15}$; le personnage central est Maximilien de Habsbourg, qui s'est proclamé empereur du Mexique. Dans Paulus unter den Juden le tyran lointain est Caligula, dans Das Reich Gottes in Böhmen, c'est Sigismond de Luxembourg, originaire de Bohême, mais devenu despote étranger, puisque empereur d'Allemagne et ennemi des Hussites. Maximilien mis à part, les monarques restent invisibles. Mais Werfel aligne dans ces pièces les représentants des classes possédantes qui soutiennent le pouvoir. Il y a partout collusion entre «Die Reichen und das Reich» ${ }^{16}$. 
8 C'est contre eux que les héros vont engager la lutte. Mais cela ne donnera pas des pièces à thèse révolutionnaire. Tout va se jouer entre deux catégories de révolutionnaires, les extrémistes et les réalistes, selon le schéma suivant:

\begin{tabular}{|l|l|l|l|}
\hline & oppresseurs & $\begin{array}{l}\text { révolutionnaires } \\
\text { extrémistes }\end{array}$ & Révolutionnaires réalistes \\
\hline Spiegelmensch & Ananthas et ses monstres & Spiegelmensch & Thamal \\
\hline $\begin{array}{l}\text { Bocksgesang } \\
\text { Schweiger }\end{array}$ & $\begin{array}{l}\text { les Turcs } \\
\text { Viereck }\end{array}$ & $\begin{array}{l}\text { Juvan } \\
\text { les sociaux-démocrates }\end{array}$ & $\begin{array}{l}\text { l'homme-bouc } \\
\text { Schweiger }\end{array}$ \\
\hline $\begin{array}{l}\text { Juarez u.M. } \\
\text { Paulus unt.d.J. } \\
\text { Das Reich Gottes }\end{array}$ & $\begin{array}{l}\text { les monarchistes } \\
\text { les Romains }\end{array}$ & $\begin{array}{l}\text { Maximilian } \\
\text { lesoisés }\end{array}$ & $\begin{array}{l}\text { Juarez } \\
\text { Paul } \\
\text { Julian Cesarini }\end{array}$ \\
\hline
\end{tabular}

9 À l'encontre des interprétations passées, il devient donc possible d'avancer un nouvel éclairage de la pièce Bocksgesang: l'homme-bouc n'y est nullement le symbole du Mal comme les montres de Spiegelmensch, mais bien le symbole de la déréliction et de toutes les misères humaines. Au lieu de l'accepter comme un frère, les paysans en révolte se le donnent pour roi et en font seulement alors un monstre abominable. Le peuple abusé se crée des êtres fantastiques, vampires et homme-bouc, ou bien le bouc émissaire dans Paulus unter den Juden, et cela les détourne de leurs vrais ennemis et de la lutte révolutionnaire.

De même, dans Juarez und Maximilian, la critique n'avait pas vu que, contrairement au schéma historique scolaire, Juarez est le révolutionnaire victorieux devenu homme de raison et de modération, et que c'est Maximilien qui est l'intellectuel extrémiste, coupé des réalités, qui veut brûler les étapes du socialisme. Il n'en tient pas moins à son titre d'empereur, tout comme ses homologues des autres pièces, lesquels nourrissent tous des prétentions de chefs.

\section{Le théâtre des années vingt comme contribution à la «Neue Sachlichkeit»}

11 Désaveu de l'extrémisme: c'est d'abord cela, la Neue Sachlichkeit chez Werfel. Les héros révolutionnaires désavoués sont des hommes-miroirs livrés à l'illusion. N'acceptant pas les réalités, ils les regardent dans le miroir, où ils tiennent à voir au premier plan leur propre image. Ce faisant, ils inversent littéralement la réalité. Ils n'acceptent évidemment pas les autres, mais veulent se voir en eux. Comme ils ne s'y rencontrent pas, ils veulent combattre et détruire ce qui se refuse à cette accommodation. Juarez dit justement de Maximilien : "Cet homme se regarde dans un miroir $\aleph^{17}$, et Max réclame encore un miroir juste avant son exécution! Les héros objectifs, au contraire, sont ennemis des miroirs. Paul se sépare du zélote Chanan en disant : "Je ne voudrais pas continuer à me regarder en toi comme dans un miroir " ${ }^{18}$. Dans le lyrisme et la prose de Werfel, le thème du miroir est insistant et toujours hostile. L'image inversée qu'il donne est une atteinte à l'intégrité du Moi. On a pu 
choisir le miroir comme critère thématique pour distinguer Expressionnisme et Neue Sachlichkeit : le Spielgelmensch de Werfel serait caractéristique de l'expressionnisme et le miroir démythifiant à la fin du Hauptmann von Köpenick (1930) servirait à définir la Neue Sachlichkeit ${ }^{19}$. Irréalistes, peu avares du sang de leur prochain, les hommesmiroirs de Werfel donnent tête baissée dans les provocations du pouvoir. Ils sont «les alliés objectifs de la réaction ». Le gouverneur Marullus avoue tout crûment : « Je suis le plus empressé promoteur d'une révolte juive, que je veux écraser, mais non point empêcher $»^{20}$.

12 Les trois premiers héros positifs, toutefois, Thamal, l'homme-bouc et Schweiger, n'ont aucune efficacité politique. Nous sommes dans la phase de transition entre Expressionnisme et Neue Sachlichkeit. En 1930, Alfred Heuer explique à ce propos : « De même que Dürer, maître de deux mondes, se plaçait exactement entre période gothique et Renaissance, l'ancien et le nouveau se combattent aujourd'hui aussi dans la poitrine de chaque artiste». Et d'opposer dans le cas de Werfel Spiegelmensch à Der Abituriententag. "C'est pourquoi, poursuit Heuer, je rejette le terme de "Nouvelle objectivité" (Neue Sachlichkeit). Parlons donc de "Réalisme magique" (Magischer Realismus), d'une "objectivité transcendantale" (Transzendentale Sachlichkeit), selon le mot du peintre Beckmann! $»^{21}$. À cause de leurs moyens scéniques, on peut appliquer aux trois premières pièces des Années Vingt de Werfel le terme de "Réalisme magique ", qui est une composante de la Neue Sachlichkeit en peinture ${ }^{22}$. Werfel alors crée en expressionniste pour dénoncer l'expressionnisme. Ce porte-à-faux entre retour au mythe et drame moderne explique l'échec de ces trois premières pièces à la scène ${ }^{23}$. Werfel lui-même voulut faire mieux. Dans un journal de 1923, il note le rapport "souterrain " qui relie Bocksgesang, Schweiger et les Beschwörungen, recueil lyrique de 1923, lequel baignait "dans un monde de démons aux niveaux inférieurs", et il ajoutait : «Mon prochain livre doit dépasser cela $»^{24}$. Ce prochain livre sera Juarez und Maximilian, " pièce où j'ai pris mes distances $»^{25}$. Werfel découvre la matière historique comme la meilleure voie pour progresser dans l'objectivité. Et avec Juarez und Maximilian, de 1924, il se retrouve en plein dans la Neue Sachlichkeit. 1924 est aussi le repère choisi par Lethen comme début de ce courant ${ }^{26}$. Les amples vues historiques de Juarez, Paulus et Das Reich Gottes sont caractéristiques de la Neue Sachlichkeit. Seul Juarez obtint le succès auprès du public, sans que ce succès ne soit répercuté par la critique. Werfel avait donné à ce premier drame historique le sous-titre: "Dramatische Historie » - tableaux historiques sous forme dramatique. Cela dérouta la critique. "La relation entre "drame" et "genre épique" se heurta à la désapprobation $\aleph^{27}$. Il y eut au moins Herbert Ihering pour tenter de démêler cet enchevêtrement. Il avait peu apprécié Spiegelmensch, Bocksgesang et Schweiger ${ }^{28}$. Dans sa critique de Juarez, il trouve que cette pièce est «le plus vrai, le plus chaleureux, le plus humain, le meilleur » des drames de Werfel. Néanmoins, il pense que l'intention de l'auteur a échoué : «L'histoire dramatique de Werfel n'appartient pas à la forme intermédiaire entre récit épique et drame, mais à la forme située entre roman et théâtre. Des trouvailles romanesques sont transposées sur le plan théâtral. Il laisse l'antagoniste républicain, le citoyen-président Juarez, derrière les coulisses, il fait se rapporter tous les événements à lui, il le fait agir et contre-attaquer invisiblement, mais jamais il ne le montre. Seulement, c'est justement cette idée qui devrait condenser l'action, donner de l'élan aux péripéties, donner du raccourci à ce qui est ample. C'est justement cette idée qui devrait accentuer le romanesque dans le sens dramatique, le scinder en fonction du théâtre. Cela ne se produit pas $»^{29}$. 

les « défauts » de son drame le fait que "l'antagonisme intérieur caché ne soit pas tout à fait dégagé. Le phénomène abstrait qu'est Juarez n'est pas complet $\aleph^{30}$. Il se dit également insatisfait de Paulus ${ }^{31}$. Le problème est celui de la Neue Sachlichkeit. Werfel veut délivrer un message de morale politique, il exige de ses héros qu'ils concilient les impératifs d'humanité et de lucidité politique, qu'ils se montrent hommes de raison et de modération jusqu'à mortifier leur personnalité. Juarez agit efficacement, mais si modestement qu'il n'apparaît pas sur scène. Paul n'est plus le zélote Schaul, car il estime que la Révolution a déjà été faite par le Rabbi Jeoschua ${ }^{32}$. Le cardinal Cesarini, ancien persécuteur des Hussites, se met à négocier avec eux, les invite au Concile de Bâle où il prend même leur défense. Autrement dit, le héros politique de Werfel est dépourvu de dimension tragique.

Sur le plan de l'esthétique, Werfel se rapproche de la « Nouvelle Objectivité » en se distançant de l'expressionnisme. Certes s'il le fait, ce n'est pas tout de suite au nom de l'objectivité. Immédiatement après Spiegelmensch, il commence par reprocher à la dramaturgie moderne de nier tous les effets «magiques» du théâtre. Pour lui, l'expressionnisme est «une adoration effrénée de la technique du sketch et du cinéma ", qui détruit le discours dramatique en le réduisant à quelques mots inducteurs proférés sur un ton extatique ${ }^{33}$. Mais il publie aussi une « Dramaturgie et interprétation du spectacle scénique "Spiegelmensch" $\$^{34}$. Le chapitre IV de cet important essai propose l'ironie comme base de l'effet théâtral :

Savoir que nous rêvons... voici la plus concrète définition de l'ironie. (...) L'effet est la morale du dramaturge (...). Le niveau de conscience du monde scénique ne doit pas être identique avec le niveau de conscience du monde des spectateurs ${ }^{35}$.

Le spectateur doit en savoir plus que les personnages sur scène ${ }^{36}$. Walter Sokel peut donc démontrer que ce texte fit date en dépassant effectivement l'attitude expressionniste, dans laquelle « toutes les figures et tous les événements du drame sont présentés dans la perspective du personnage principal » et qui, de plus, tend à la "totale identification du spectateur avec la perspective de ce personnage $\|^{37}$. Werfel était assez lucide pour reconnaître que sa production de jeunesse se rangeait dans l'expressionnisme. Dans une esquisse d'autobiographie datant de 1921-1923, il rappelle qu'il fut lui aussi prisonnier de "l'attitude artistique devant la vie qu'on a pris l'habitude d'appeler expressionnisme». La définition qu'il en donne revient à une confusion entre théâtre et poésie ${ }^{38}$. Au contraire, dans sa "Dramaturgie» de Spiegelmensch, il précisait : «Le théâtre est la maison du comédien et non pas celle du poète $»^{39}$. Dans cet essai et dans son journal de 1921, il voit dans l'expressionnisme un profond divorce entre art et réalité, un "Snobisme» qui épouse cyniquement une perversion due à l'époque ${ }^{40}$. Après sa « Dramaturgie » il restera huit ans sans écrire de grand essai. Le prochain sera justement le pamphlet Der Snobismus als geistige Weltmacht, où il préconise plus de respect de l'art envers les réalités ${ }^{41}$.

16 Évidemment, Werfel a eu du mal à liquider l'expressionnisme. Il est comparable à Brecht et Musil, tous deux adversaires de l'expressionnisme, bien qu'ils en aient été tributaires à leurs débuts. D'ailleurs, au moment où Brecht et Werfel se démarquent de l'expressionnisme en formulant une théorie dramatique, ils prennent exactement la même voie: "En exigeant ainsi que l'auteur soit omniscient en ce qui concerne sa création, le jeune Brecht se rapproche de la théorie werfelienne des perspectives, et 
plus mûrit en lui, au cours des Années Vingt, l'idée du théâtre épique, et plus ce rapprochement s'accuse ${ }^{42}$.

Mais peut-on compter Werfel parmi les auteurs de la Neue Sachlichkeit, alors que dans ses «Positions de principes » sur le théâtre, il continue à défendre la part du rêve contre «le naturalisme gâteux» ?43. Justement, la tragédie historique a pour lui le grand mérite de réconcilier rêve et réalité ${ }^{44}$. Il écrit dans son Argument de Paulus unter den Juden:

De même que le rêve est la reconstitution en profondeur de la vie personnelle, la tragédie est la reconstitution en profondeur des événements mondiaux ${ }^{45}$.

Dans l'essai Historisches Drama und Gegenwart, écrit à la suite de Das Reich Gottes in Böhmen, il n'abandonne pas son principe : « Le théâtre est du rêve », et d'ajouter :

Depuis qu'existe l'Histoire, la situation originelle des luttes de pouvoir et d'idées de l'homme ne s'est pas modifiée: Richesse-Pauvreté, Despotisme-Esclavage, FoiCritique etc.. Un fait d'Histoire bien délimité, définitivement cristallisé, tel que veut le représenter la tragédie historique, a la puissance de la vérité, car il s'est vraiment produit et possède la force du symbole, en se rapportant à notre situation, dans le sens d'une clarification ${ }^{46}$.

La démarche a parfaitement réussi. Car les drames historiques de Werfel débordent largement leurs cadres historiques. Ils présentent non seulement des révolutions politiques, mais aussi des révoltes coloniales. Juarez pose avec vingt ans d'avance le problème de décolonisation: un million de Blancs face à neuf millions d'Indiens ${ }^{47}$. "César, dans sa divinité, proclame Marullus, ne connaît que des populations et non des peuples $»^{48}$. L'erreur des héros activistes est de se limiter soit au problème social, tel Maximilien, soit au problème national, comme Chanan, ou de se tourner tantôt vers l'un, tantôt vers l'autre, comme Prokop, au lieu de comprendre que la solution des deux questions doit être liée par la politique. Maximilien ne voit que «le peuple indien », là où Juarez voit déjà « des Mexicains $»^{49}$. Dans le manuscrit de Werfel à la Bibliothèque Municipale de Vienne on lit d'ailleurs à cet endroit "prolétariat " à la place de " peuple». L'auteur a opéré la modification sans doute à cause de l'incidence trop nettement politique du terme de prolétariat. Car Maximilien refuse justement la politique, qui serait l'appréhension simultanée des solutions sociale et nationale. «Je détruis la politique ", proclame-t-il et il veut " libérer le pays de la politique $»^{50}$. Il ne se veut pas chef de parti, mais ne se rend pas compte qu'il est le jouet des monarchistes, ce que sait voir son antagoniste Porfirio Diaz, porte-parole de Juarez ${ }^{51}$.

Donc l'attitude de Werfel a évolué. Il n'adhère toujours pas à l'action politique, mais il accepte implicitement le fait politique. Seulement, il ne faut pas vouloir être à la fois un politique et un idéaliste. Il le dit explicitement dans ses commentaires de Das Reich Gottes in Rohmen: "Je crois maintenant que tout politicien idéaliste est condamné à échouer $»^{52}$.

21 Comme toutefois il ne s'agit nullement d'un théâtre à thèse, il a provoqué certaines déceptions. Après lecture des premiers extraits de Juarez à Berlin (dans le «Bürgersaal » de l'Hôtel de Ville), le Vorwärts se fit l'écho de la déception des sociaux démocrates: "On ne pouvait pas reconnaître dans quel sens se dirige la tendance du drame. Mais il semble presque que Werfel veuille corriger l'Histoire, comme s'il se trouvait du côté de l'empereur et donnait tort au révolutionnaire Juarez. Il serait dommage que Werfel, avec son grand talent, donne dans de tels errements ${ }^{53}$. En réponse, Werfel se montra 
fier du malentendu, qui prouve qu'il a «satisfait à une loi essentielle de l'art tragique : la justice $»^{54}$. Sa réponse à une interview de la Prager Presse en 1930 dit la même chose.

J'écris sans le vouloir, toujours à rencontre des pièces à thèse, des pièces à antithèses. Cela est inséparable de ma foi dans le drame comme forme dialectique

(...). Il va de soi que les deux contraires doivent avoir raison et font appel à la même sympathie ${ }^{55}$.

La nouvelle mentalité objective demande l'humilité morale, qui consiste à mettre en veilleuse la passion du bien. "Quelque chose de bon, demande un protagoniste de Juarez, peut-il être bâti sur une erreur de calcul ? ${ }^{56}$. Porfirio Diaz semble définir la Neue Sachlichkeit quand il affirme : "Juarez ne fait pas le bien, mais il fait ce qu'il faut, et c'est cela seul qui est bon par ses conséquences ${ }^{57}$.

Tous les personnages véhiculent une part de vérité, même les guerriers et les dirigeants : «La bonne volonté, Sire, dit Bazaine, est le plus souvent de la mauvaise politique $»^{58}$. Même le Capitaine Pierron, spécialiste des méthodes anti-guerilla, a de fortes paroles: "Toute faiblesse est doublement inhumaine, car elle retarde la consolidation et prolonge l'effusion de sang $»^{59}$.

En 1988 une étude de Axel Schalk place les drames historiques de Werfel, Juarez und Maximilian en tête, dans la Neue Sachlichkeit, montrant toute la nouveauté qui consiste à affronter le héros Max à un antagoniste complètement absent de la scène. "La confrontation traditionnelle héritée de l'ancien drame historique - Maria contre Elisabeth ; Tell contre Geissler - n'a pas lieu (...) Maximilien combat un fantôme, une idée. Là réside la performance dramatique de Werfel, qui en même temps critique l'ancien drame bâti sur un antagonisme $"^{60}$. L'appartenance des drames de Werfel à la Neue Sachlichkeit est désormais indiscutable. Le dernier volume des Rowohlt Bildmonographien, consacré à Werfel, en fait également état ${ }^{61}$.

\section{La Neue Sachlichkeit dans le récit}

« Pour l'essentiel, le système dramatique du théâtre de la Neue Sachlichkeit admet une définition négative, puisqu'il se conçoit par rapport à un état plus ancien du drame, dont il veut se démarquer. Les traits caractéristiques de ce démontage des conceptions dramatiques libérales seront résumés sous les rubriques suivantes : réalité sans forme, conflit sans légitimité, héros sans pathétique, discours sans expression ni réponse (...). Le désordre de l'événement véritable n'étant guère maîtrisable, il s'exprime, en terme dramatique, dans une série de scènes brèves qui souvent s'alignent sans enchaînement " 62. Cela s'applique tout à fait au théâtre de Werfel des Années Vingt. L'ampleur de l'action, la prétention à l'exactitude historique, la pesante fidélité aux sources et jusqu'à la minutie dans la reconstitution des costumes, le réalisme enfin qui a nui à l'audience des pièces, poussaient Werfel vers le roman ${ }^{63}$.

Au début des Années Vingt, il avait déjà écrit une longue nouvelle, Nicht der Mörder, der Ermordete ist schuldig (1920) et un roman biographique, Verdi (1923). Ce sont deux œuvres expressionnistes. Dans la première, l'imagination du héros lui fait perdre de vue les réalités. Il passe à l'action révolutionnaire non pas par solidarité avec les opprimés, mais par haine de son père, dont il s'exagère la dureté. Quant à Verdi, il décrit une crise de productivité du héros et l'opposition entre deux révolutionnaires de la musique, Wagner et Verdi. Qui l'emportera dans la conquête de l'art populaire ? Ce sera Verdi, car il finira par sacrifier à la simplicité et à la spontanéité sa subjectivité et son 
besoin de grandeur. Renonçant à l'art cérébral qui le fascine et le repousse chez son rival, il adopte pour maxime: "Que rien ne vienne d'un labeur ingénieux!» («nicht erkklügeln!» ${ }^{64}$. Walter Sokel considère le Verdi de Werfel comme l'apogée et le point final de l'expressionnisme ${ }^{65}$. On constate donc que tout comme les trois premiers drames des Années Vingt, ces deux œuvres narratives de style expressionniste portent aussi condamnation de cette mentalité. D'ailleurs, à chaque fois que Werfel parle de l'expressionnisme, c'est soit pour lui opposer Verdi, soit pour rappeler que son amour pour Verdi est allé de pair avec ses premiers élans expressionnistes ${ }^{66}$. Il s'étend pour la première fois sur l'antinomie Wagner/Verdi... dans l'essai sur la dramaturgie de Spiegelmensch !67

Après Das Reich Gottes in Böhmen, la grande veine dramatique de Werfel se rétrécit. Dans le reste de son œuvre, ce sont les romans qui occupent la place marquante. Et de même que les nouvelles de jeunesse sont - logiquement sinon chronologiquement - la charnière entre lyrisme et théâtre, c'est une série de nouvelles qui, en 1927, entre Paulus et Das Reich Gottes, va servir à réorienter la production de Werfel. L'année 1927 est chez lui l'année de la nouvelle par excellence. Il en écrit six, qui sont des chefsd'œuvres: Die Entfremdung, Geheimis eines Menschen, Die Hoteltreppe, Kleine Verhältnisse, Der Tod des Kleinbürgers, Das Trauer haus ${ }^{68}$. C'est à cause de ces nouvelles que la monumentale histoire de la littérature autrichienne de Nagl-Zeidler-Castle classe expressément Werfel dans la Neue Sachlichkeit ${ }^{69}$. En effet, ces nouvelles prennent sans phrases leur parti du déclin de l'ancienne Autriche: «Rien ne meurt si l'heure n'en a pas sonné $»^{70}$. À la même époque, Félix Bertaux cerne parfaitement l'optimisme lucide de la Neue Sachlichkeit: "Il est des choses qui se défont parce qu'elles étaient mal faites, et il faut les refaire avec un courage de l'esprit que n'impliquent ni les revanches, ni les restaurations. Tout est redevenu possible pour une jeunesse qui a assisté à l'impossible, et n'éprouve pas la surprise de ses ascendants devant leurs catégories renversées $\aleph^{71}$. Les nouvelles de Werfel dénoncent l'aliénation, mais ne préconisent pas non plus une révolution ni une émancipation provocatrice. Les seules femmes équilibrées et qui ne fassent pas pitié dans ce groupe de nouvelles sont... les prostituées de Das Trauerhaus. Ces récits peuvent être considérés comme le pendant autrichien des descriptions sociales de Fallada.

À ces nouvelles fait suite en 1928 Der Abituriententag. Cette œuvre est en fait une articulation entre nouvelle et roman, mais elle relève de l'esthétique de la nouvelle. Elle restitue une histoire de jeunesse, la brusque déchéance d'un lycéen modèle en butte à l'envie de ses camarades. L'événement est brutal, raconté avec froideur et logique. Pourtant le narrateur, qui se souvient à vingt-cinq ans de distance du rôle peu glorieux qu'il joua alors, baigne dans les interrogations. Devenu procureur, il croit, sans aucune certitude, reconnaître sa victime de jadis dans le suspect de crime qu'on lui présente. Il écrit l'aventure du lycée sous le coup d'une trompeuse lucidité nocturne, à coups de tasses de café. Mais son manuscrit reste indéchiffrable; inconsciemment et pour se disculper, le magistrat fait tout pour se persuader qu'il a rêvé. L'âge des personnages, le milieu du lycée, rappelle Nicht der Mörder, der Ermordete ist schuldig. Une comparaison détaillée entre les deux nouvelles pourrait servir à définir par contraste les thèmes et le style de l'expressionnisme et de la Neue Sachlichkeit. Dans Der Abituriententag, les motivations profondes des héros sont bien mieux cachées que dans le Mörder. "Tout aussi variés que sont les événements, écrit Richard Specht, tout aussi singulier est l'enchevêtrement des motivations psychiques", Werfel "ne s'immisce jamais, même pas en tant que psychologue, alors qu'il l'est ici plus que jamais auparavant ${ }^{72}$. Un autre 
critique introduit son compte rendu en louant la «maîtrise souveraine dans la technique psychanalytique " dont Werfel a fait preuve et d'ajouter: "peut-être la raison en est-elle la nouvelle orientation dramatique de Werfel, mais peut-être réside-telle aussi dans l'objectivité, dont on parle tant $»^{73}$. La différence principale en effet est que les héros de Der Abituriententag n'ont pas de pères à affronter pour se libérer, ce qu'a bien vu Jost Hermand dans son article « Édipus lost », où il examine en priorité le Fabian d'Erich Kästner: "Au contraire des "fils" expressionnistes, il n'a rien eu à "surmonter", ce qui l'aurait forcé à édifier sa propre image du monde, mais dès le départ, il se laisse passivement dériver (...). Une façon analogue d'appréhender la vie est représentée dans les romans "sans pères" tels que Joseph sucht die Freiheit de Hermann Kesten (1927), Der Abituriententag de Franz Werfel (1928) et Der Schüler Gerber hat absolviert de Friedrich Torberg (1930), dont le caractère fondamental, axé sur la Neue Sachlichkeit, est en grande partie conditionné par l'absence d'orientation intérieure de cette "génération perdue" $\gg^{74}$.

Cette génération « objective » évacue par ailleurs le problème de la faute, en regardant les réalités avec un œil froid, justement avec la froideur morale d'Adler, la victime dans Der Abituriententag. Celui-ci montre au futur procureur Sebastian son essai sur «L'Homme comme jouisseur et objet de jouissance ». Les animaux supérieurs, écrit-il, ne se mangent pas entre eux, au contraire de l'Homme :

L'Homme ne se distingue pas du reste de la nature par la raison, mais par l'appétit (...). Les cannibales ne mangent pas de la chair humaine parce qu'ils y sont forcés, mais par gourmandise, car en effet l'être humain est le plus épicé parmi les meilleurs morceaux ${ }^{75}$.

la Neue Sachlichkeit. Ses drames Paulus unter den Juden et Das Reich Gottes in Böhmen relèvent par certains côtés de l'histoire des religions. Mais ni ceux-là ni les autres ne sont de structure religieuse. La première inspiration de Paulus fut une idée de drame lue dans la revue Pan vers 1913-1914... dans laquelle Paul aurait assassiné Jésus ! ${ }^{80}$ Quant à Das Reich Gottes, il a été induit... par des lectures marxistes ! ${ }^{81}$ Et tous les héros négatifs son rival en refroidissant l'enthousiasme de Sebastian pour le Cercle de Lecture fondé par celui-ci et qui permet à Adler de se distinguer. Sebastian, le futur procureur, laisse sa conscience s'émousser par trop de sentimentalisme, mais Adler pèche par l'excès inverse : «Il voyait des larmes en général, une souffrance, une âme, pas Sebastian, pas moi, être dénaturé qu'il était à force d'abstraction $»^{76}$. Et Adler abdique tout de suite devant la violence. Pour une raison futile, après une empoignade où Sebastian l'a vaincu de peu, il se laisse complètement dominer. L'intelligence recule devant la force. Pourtant, la froide objectivité d'Adler, il aurait fallu l'assumer et même l'aimer, car elle était vision de la réalité. Elle aurait pu contrebalancer salutairement les vanités et les emballements fumeux des autres élèves. L'auteur aide le lecteur en lui donnant la solution morale en exergue de la nouvelle et dans le corps du texte. C'est la maxime de Goethe : «Contre les avantages d'un autre, il n'est de sauvetage que l'amour ${ }^{77}$.

les dons répartis par Dieu "dans son insondable façon de dérouter les calculs " ${ }^{78}$ Sebastian devait s'incliner devant la supériorité d'Adler : «C'était à lui, que Dieu avait donné sa grâce, et non pas à moi ${ }^{79}$.

On peut dire que par ses récits, Werfel occupe un «créneau » religieux à l'intérieur de à Das Reich Gottes, il a été induit... par des lectures marxistes !̊ Et tous les héros négatifs 
de ces drames des Années Vingt se rendent justement détestables en s'arrogeant des missions divines.

En revanche, le roman de 1927 Barbara oder Die Frömmigkeit montre que l'humble ferveur religieuse est le seul point fixe possible au milieu des tempêtes de l'Histoire. Il ne s'agit cependant pas que d'édification. Werfel souligne quelle tentation représentent le capitalisme et la technique en passe de devenir des religions de remplacement. Le personnage juif Alfred Engländer, en plein désarroi moral, religieux et mental, profère une litanie qui rend parfaitement la tension littéraire de la Neue Sachlichkeit :

Saint Capital, prends pitié de nous !

Saint Taux d'Intérêt, légitimement né de lui, prends pitié de nous!

Saint Esprit de Profit de l'Intelligence, Troisième Personne, prends pitié de nous !

Vous les très saints chœurs et prêtres du pétrole, du coton, du charbon, du cuir, de

la production de caoutchouc, de la construction mécanique, intercédez pour nous !

Tous les apôtres et évangélistes du marché de l'argent et de la science moderne en lui et par lui, intercédez pour nous!

Vous tous les saints moines et ermites des trusts et sociétés par actions dans vos résidences de campagne avec eau courante, chauffage central et tableaux de Rembrandt, priez pour nous $!^{82}$

On n'a pas à faire ici à une ironie à bon marché dirigée contre les valeurs modernes. Le personnage prie vraiment ces forces de notre temps de tourner au bien et non au mal de l'humanité. En effet, Barbara n'est pas un roman réactionnaire ni même conservateur. La description de la fin de la Double Monarchie n'y est pas nostalgique. Werfel approuve pacifisme et antimilitarisme, célèbre la Révolution et la discipline des masses populaires envahissant le Ring en novembre 1918, salue les chefs de la socialedémocratie et leurs réalisations. Mais il met aussi en garde contre le chauvinisme des nationalités émancipées, contre toute violence, même quand elle se dit révolutionnaire. Nicht der Mörder... dénonçait la fausse solidarité avec les déshérités qui n'était que haine pour le milieu privilégié où avait grandi le héros. Barbara dévoile la duplicité d'intellectuels qui, faute d'avoir connu les champs de bataille, réclament du sang dans l'ivresse de la Révolution ${ }^{83}$.

Sous une forme achevée et à douze ans de distance, Werfel répète donc l'avertissement amorcé dans son fragment Stockleinen. En 1928, il avait même donné une lettre ouverte à la revue nationaliste Der Vormarsch, où il déclarait condamner aussi bien les attentats de la gauche que ceux des conservateurs. Il s'en prenait spécialement à la presse communiste qui ne savait flétrir que la violence de ses adversaires politiques ${ }^{84}$. Malheureusement, cette déclaration n'a pas été reproduite dans le volume d'essais de Werfel.

\section{Actualité de Werfel critique de l'idéalisme}

De la profonde réflexion de Werfel, de sa modération, de ce qu'il faut bien appeler son réalisme en politique, la critique marxiste de l'époque n'a retenu que sa méfiance envers la Révolution. Exactement comme l'exprime le sous-titre du livre de Lethen, elle a identifié la Neue Sachlichkeit à la Werfel à un « Socialisme blanc ». Dès 1921, Die Rote Fahne, organe du Spartakusbund de Karl Liebknecht et Rosa Luxembourg, avait constaté que les expressionnistes n'étaient pas les alliés des marxistes, à cause de leur intellectualisme, de leur ignorance des masses laborieuses et de leur combat uniquement en faveur de l'individu et non pas pour changer les réalités extérieures ${ }^{85}$. 
Quelques années plus tard, Piscator avoue posséder Werfel dans sa bibliothèque, mais dans un entretien avec Herbert Ihering il se garde bien de prendre la défense de Werfel, vu que celui-ci rejette le théâtre à thèse. Ihering argumente : «Samedi dernier, la pièce de Werfel, réussie du point de vue de la forme, mais fragile, Paulus unter den Juden aucun effet. Dimanche l'œuvre documentaire d'Erich Mühsam Sacco et Vanzetti impression bouleversante. La différence est une preuve ${ }^{86}$. On en n'est pas encore à désigner Werfel comme allié de la bourgeoisie. Mais dès 1930, de même que les milieux sociaux-démocrates s'étaient mépris sur Juarez und Maximilian, Ihering dénonce dans Barbara " l'insidieuse réaction qui approche de toutes parts " ${ }^{87}$. En 1931, après parution de l'essai de Werfel Realismus und Innerlichkeit, qui renvoyait dos à dos capitalisme et bolchevisme, les fronts sont nets. Die Rote Fahne, Internationale Literatur, Die Linkskurve attaquent Werfel ${ }^{88}$. En 1934, l'essai de Lukács Grösse und Verfall des Expressionnismus condamne le «pacifisme factice » de l'époque expressionniste en des termes qui visent à coup sûr la non violence de la Neue Sachlichkeit ${ }^{89}$. Dans un autre essai de 1934, Lukács adresse aux naturalistes exactement le même reproche qu'à l'expressionnisme et pense aussitôt à Werfel : «De plus en plus les exploiteurs et oppresseurs sont représentés comme "victimes de la nécessité", on "se met à leur place", "comprend" leur situation et la nécessité de leur comportement, les entoure de la sympathie mélancolique pour le "tragique" qui fait qu'ils n'ont pas le choix. Cette répercussion idéologique, apologétique de la "capacité de sentir" (Einfühlung) comme méthode créatrice dépasse largement le naturalisme pour se poursuivre avec Rilke et Werfel $»^{90}$. C'est reconnaître implicitement chez Werfel une dimension réaliste. C'est vrai que Werfel excuse les aristocrates, eux aussi étant victimes de l'aliénation qui les empêche de penser autrement. Maximilien est "victime de sa naissance $»^{91}$. "Seule la mort, dit Klenau, aristocrate égaré chez les Hussites par amour de la femme de leur chef, peut effacer par sa brûlure la qualité d'aristocrate $»^{92}$. Il est comme forcé de trahir : « Un seigneur reste de toutes façons un seigneur... $»^{93}$.

En 1938, Ernst Bloch défend contre Lukács l'authenticité du pacifisme werfelien de la période expressionniste, mais il accorde : "C'est vrai, Werfel et d'autres de son genre ont transformé après la guerre leur pacifisme abstrait en une trompette d'enfant; le mot d'ordre «non-violence" est ainsi devenu, vis-à-vis de la Révolution, un mot d'ordre anti-révolutionnaire $»^{94}$.

Julius Bab, d'ailleurs, voyait dans la Neue Sachlichkeit tout simplement un retour au naturalisme ${ }^{95}$. La grande différence est que pour Werfel la dimension transcendante fait partie des réalités. Il existe aussi un extrémisme de l'objectivité, par exemple dans le choix opéré par Brecht pour couronner un poète de notre temps. La Literarische Welt avait organisé en 1926, à l'occasion du premier anniversaire de sa fondation, un concours littéraire, le jury se composait de Döblin pour le roman, de Ihering pour le théâtre et de Brecht pour le lyrisme. Or ce dernier décréta qu'aucun poète candidat ne méritait le prix. La raison : ce sont tous les imitateurs de George, Rilke, Werfel, « dont il fait peu de cas ", à cause de leur orientation bourgeoise ${ }^{96}$. On savait par d'autres notes de Brecht qu'il reprochait à Werfel son «Kitsch», son «indigence démocratique »... et son succès !97 Lors du concours de 1926, Brecht opte pour Hannes Küpper, lequel n'était même pas candidat. Brecht a trouvé ses poésies dans un journal sportif. Chez Küpper, l'homme de notre temps adhère aux machines qu'il dessert, il est un organisme de câbles, de tringles et de rouages ${ }^{98}$. 

«Idealistische Sachlichkeit» et la «Radikale Sachlichkeit»99. C'est cette dernière que Werfel répudie dans son essai de 1931 Realismus und Innerlichkeit et pour laquelle il forge le terme de «Radikaler Realismus» ${ }^{100}$. En 1932, son discours à la mémoire de Schnitzler contient un passage polémique contre la politisation du théâtre. ( «Je suis convaincu que quelqu'un qui veut vraiment connaître la vie des mineurs n'ira en aucun cas au théâtre à cette fin ") ${ }^{101}$. Il avait déjà désavoué l'expressionnisme dont il était pourtant le coryphée. Il n'est pas le premier artiste à se distancer d'une étiquette qu'il a pourtant contribué à frapper. Ce faisant, il ne rejette que les excès. Il se garde de rejeter le réalisme en bloc $^{102}$. Sur le plan de l'art, il devient lui-même un réaliste aux procédés narratifs traditionnels. Il doit même quelque chose à Zola, qu'il admire presque sans réserve et auquel il a consacré un essai ${ }^{103}$.

Dès sa jeunesse, Werfel appréciait chez les écrivains leur souci de «morale politique » 104 et sa critique de la Révolution, venant d'un auteur qui se voulait au départ révolutionnaire, fait de lui un précurseur des Anouilh, Bernanos, Camus, Malraux, Montherlant, Sartre. Bien des formules de son théâtre anticipent celles des Français. Des personnages lucides de Juarez und Maximilian et Das Reich Gottes in Böhmen avertissent: "Pour tout idéaliste sonne l'heure où il peut devenir ou devient un criminel »; il n'y a «jamais qu'une espèce d'exterminateur de la pire cruauté : les idéalistes $»^{105}$. Werfel combat aussi le Romantisme comme un idéalisme qui fait fi des réalités ${ }^{106}$. Cela le rapproche de façon frappante des « Nouveaux Philosophes » français, et singulièrement d'André Glucksmann et de Bernard-Henri Lévy. Comment ne pas penser aux Maitres Penseurs (1977) et au Silence on tue (1986) de Glucksmann en lisant Der Snobismus als geistige Weltmacht et Die vierzig Tage des Musa Dagh de Werfel? Il y a tout spécialement des convergences entre les vues de Werfel et Le Testament de Dieu de Bernard-Henri Lévy. Tous deux appellent romantisme la source des religions de remplacement qu'ils rejettent ${ }^{107}$. Ils voient dans la politique inhumaine l'idéalisme extrême ${ }^{108}$. Dès 1917, dans ses essais Fragment gegen das Männergeschlecht et Brief an einen Staatsmann, Werfel voyait dans le militaire et le politicien les plus dangereux des

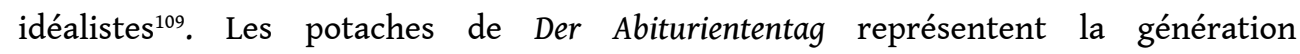
expressionniste. Contempteurs des classiques, leurs expériences cérébrales ouvrent la voie à leur cruauté mentale envers Adler. Ayant lui-même participé aux excès de la pensée et de l'art modernes, Werfel bat sa coulpe dans les Theologumena écrits sur la fin de sa vie :

Sous les rires de quelques béotiens qui jouaient à s'indigner, nous étions les minables qui commençaient à attiser la fournaise infernale dans laquelle l'humanité est en train de rôtiririo.

On a compris que la Neue Sachlichkeit n'aura été pour Werfel qu'une phase préparatoire à un message religieux. Mais qu'un Werfel ait assumé cette période prouve que la Neue Sachlichkeit est un concept solide dans l'histoire de la littérature, de l'art et des idées.

Félix Bertaux termine son Panorama de la littérature allemande en disant des auteurs des Années Vingt: "Leur froideur est désir de lucidité; leur passion, volonté; leur aventure, celle d'intelligences et de cœurs ne consentant à se donner qu'à ce qui en vaudra la peine (...). La volonté de discrimination l'emporte sur le goût des extases. 
Sous ce qui s'appelle Neue Sachlichkeit transparaît une sensibilité plus intellectuelle que celle d'hier, et gouvernée par un esprit plus libre $»^{111}$. Franz Werfel est partie prenante de ce rééquilibrage. Il fallait le souligner en cette année de son centenaire.

En 1928, Félix Bertaux avait des paroles d'espoir. À propos des écrivains de l'époque, il poursuit : «Etre Européens, ils pensent que c'est leur lot. Mais en leur for intérieur, ils nient que l'on ait jusqu'à présent trouvé la façon de l'être (...). Quelle Europe promettent ces dispositions, nul ne peut le dire (...). Une élite pressent la renaissance spirituelle de l'occident ${ }^{112}$. Ces perspectives d'alors restent d'autant plus vraies qu'elles ont survécu aux terribles épreuves. Elles sont même en voie de réalisation, puisque 1990 voit se dérouler en Europe centrale et particulièrement à Prague, patrie de Werfel, des révolutions et renaissances pacifiques, marquées de surcroît par les forces morales et religieuses que Werfel ne dissociait pas de l'objectivité ni du réalisme.

\section{NOTES}

1. - V. Félix Bertaux, Panorama de la littérature allemande, Paris, Kra, 1928, p. 254.

2. - Franz Werfel, Das lyrische Werk, Francfort/M., S. Fischer, 1967, p. 267 (Der Gerichtstag).

3. - F. Werfel, Zwischen Oben und Unten. Prosa. Tagebücher. Aphorismen. Literarische Nachträge hrsg. von Adolf Klarmann, Munich, Langen-Müller, 1975 (abr. OU), p. 207 (Fragment gegen das Männergeschlecht).

4. - Préface à Das Lied von Bernadette, toutes éditions.

5. - Bertaux, op.cit., II, 2 ; Les écrivains de choc. Passage de l'expressionnisme à l'ordre froid (p. 238-296).

6. - H. Ihering, «Der Fall Werfel», in Das Tagebuch vol. 9-10, 1928-1929, repris dans H.I., Die getarnte Reaktion. Eine Streitschrift, Berlin, 1930 et H.I., Die Zwanziger Jahre, Berlin, Aufbau Vlg. 1948, p. 195-225 ; également dans Hans Mayer Hrsg., Deutsche Literaturkritik im 20. Jahrhundert, Stuttgart, Govert, 1965, p. 640-675 (cit., p. 653).

7. - Bertaux, op. cit.., p. 248.

8. - Ibid., p. 250. Paul Fechter explique longuement le passage du lyrisme au théâtre chez Werfel : P.F., Das europäische Drama, Mannheim, Bibliographisches Institut, T. 3, 1958, p. 71.

9. - Théâtre complet de Werfel : F.W., Die Dramen, Francfort/M., S. Fischer, 1959, 21. Toutes les pièces d'avant 1920 : t. 1, p. 11-133; fragments : t. 2, p. 343-472 (abréviation pour la suite des notes : Dramen I et II).

10. - Philippe Ivernel, «L'abstraction et l'inflation tragique dans le théatre expressionniste », in : D. Bablet-J. Jacquot, L'expressionnisme dans le théâtre européen, Paris, C.N.R.S., 1971, p.91.V. aussi la thèse de Martin Arnold, Franz Werfel. Des Dichters Welt und Weg zwischen Lyrik und Drama 1910-1930.

11. - Version imprimée, Lyrisches Dasein und Erfahrung der Zeit im Früh werk Franz Werfeis, Altdorf, Huber, 1961 : « Il est préférable, à propos de ces œuvres, de parlernon de théâtre, mais de lyrisme scénique », (p. 38 et 40$)$.

Emil Staiger, Grundbegriffe der Poetik, Zürich, Atlantis Vlg., 1946, 7. Aufl. 1966, p. 154.

12. - V. Alma Mahler-Werfel, Ma vie, Paris, Julliard, 1961, p.131-132. Gina Kaus, Und was für ein Leben... Mit Liebe und Literatur, Theater und Film, Hambourg, Knaus, 1979, p. 73-74. Robert Neumann, Ein leichtes Leben, Munich, K. Desch, 1963, p. 359 et Vielleicht das Heitere, Desch, 1968, 
p. 369. Willy Haas, «Ein Dichter, nur ein Dichter». Am 10. September wäre Franz Werfel 80 geworden in : Die Welt, 10.9.1970, p. 17.

13. - V. Hans Hautmann, «F. Werfeis "Barbara oder Die Frömmigkeit" und die Revolution in Wien 1918», in Österreich in Geschichte und Literatur, n 8, 1971, p. 469-479.

14. - Principaux travaux sur le théâtre de Werfel : E. Adams-U. Kuhlmann, «Perspektiven über Werfeis dramatisches Schaffen», in : Views and Reviews ofgerman modern Literature, Festschrift für A. Klarmann, Munich, 1974, p. 195-212 ; Martin Arnold, op. cit. ; R. Broch, Die Dramen Franz Werfeis, Diss., Vienne, 1935 ; E. Hunna, Die Dramen von Franz Werfel, Diss., Vienne, 1948 ; Hanns Karlach, Werfeis Kampf um das Drame. Des Dichters theatralische Anfänge, Dipl., Prague, 1963 ; H. Meister, Werfeis Dramen und ihre Inszenierungen auf der deutschsprachigen Bühne, Diss., Cologne, 1964 ; H. Rück, Franz Werfel als Dramatiker, Diss., Marbourg, 1965 ; H. Vogelsang, Der Dramatiker Werfel, in : 136. Jahresbericht des Schottengymnasiums, Vienne, 1961, p.52-65 ; J.D.A. Warren, «Werfels's Historical Dramas», in L. Huber Hrsg., Franz Werfel. An Austrian Poet reassessed, Oxford, Berg Publishers, 1989, p. 153-173 ; P. Wimmer, Franz Werf els dramatische Sendung, Vienne, Bergland Vlg., 1973.

15. - Dramen I, p. 413.

16. - Ibid., p. 474.

17. - Ibid., p. 393.

18. - Ibid., p. 494.

19. - Reinhold Grimm, in R. Grimm-J. Hermand Hrsg., Die sog. Zwanziger Jahre, Bad Homburg, Gehlen, 1970, p. 16.

20. - Dramen I, p. 497.

21. - Alfred Heuer, "Ausdrucksform und Neue Sachlichkeit ", in Zeitschrift für Deutschkunde, vol. 44, 1930, p. 330-331.

22. - V. Wieland Schmied, Neue Sachlichkeit und magischer Realismus in Deutschland, Hannovre, 1969. En outre, on a parlé très tôt de « Nachexpressionismus », v. Franz Roh, Nach-Expressionismus. Magischer Realismus. Probleme der neuesten europäischen Malerei, Leipzig, 1925.

23. - La première de Spiegelmensch fut un succès à Leipzig le 15.10.1921, puis ce fut un échec à Stuttgart et Vienne (v. Alma Mahler-Werfel, op. cit., p. 169 et 172). Bocksgesang fut un échec, sauf en Amérique où il fonda la réputation de l'auteur (v. F. Lambasa, «F. Werfel's Goat Song», in : L.B. Foltin Hrsg., Franz Werfel, 1890-1945, University of Pittsburg Press, 1961, p. 69-82). La réaction fut exactement parallèle pour Schweiger (v. L.B. Foltin, Franz Werfel, Stuttgart, Metzler, 1972, p. 47-52 et OU p. 679-680).

24. - OU, p. 682 .

25. - Ibid., p. 688-689.

26. - Helmut Lethen, Neue Sachlichkeit, 1924-1932. Studien zur Literatur des «Weissen Sozialismus», Stuttgart, Metzler, 1970. C'est en effet en 1924 que Rudolf Kayser parle pour la première fois de «Neue Gegenständlichkeit», v. Bertaux, op. cit., p. 289.

27. - H. Meister, op. cit., p. 127.

28. - H. Ihering, Von Reinhardt bis Brecht, Berlin, Aufbau Vlg., 1961, t. 1, p. 358-359, t. 2, p. 118.

29. - Ibid., t. 2, p. 120.

30. - OU, p. 689 (Journal).

31. - Ibid.

32. - Dramen, I, p. 472 et 527.

33. - «F. Werfeis Absage an die Expressionnisten. Ein Gespräch mit dem Dichter». In: Wiener Mittagspost, Vienne, 21.5.1930-1920, p. 3. Le texte de OU, p. 591-592 n'est qu'un très court extrait de cette interview.

34. - OU, p. 222-260.

35. - Ibid., p. 230, 232, 235.

36. - Ibid., p. 269. 
37. - Walter Sokel, « Brecht und der Expressionnismus », in : R. Grimm-J. Hermand, op. cit., p. 58.

38. - OU, p. 702 (Autobiographische Skizze).

39. - Ibid., p. 232.

40. - Ibid., p. 229 (Dramaturgie und Deutung des Zauberspiels "Spiegelmensch")

et p. 673 (Journal).

41. - OU, p. 260-278.

42. - W. Sokel, loc. cit., p. 62.

43. - OU, p. 260-261 (Grundzüge).

44. - Ibid., p. 597-599 (Historisches Drama und Gegenwart).

45. - Dramen I, p. 559.

46. - OU, p. 597 (Historische Drama und Gegenwart).

47. - Dramen I, p. 404.

48. - Ibid., p. 472.

49. - Ibid., p. 455.

50. - Ibid., p. 404 et 438.

51. - Ibid., p. 426-427.

52. - «Wie mein "Reich in Gottes in Böhmen" entstand», in Freiburger Theaterblätter, 1931, p. 162-164 (pas dans OU).

53. - Vorwärts, Berliner Volksblatt. Zentralorgan der Sozialdemokratischen Partei Deutschlands, 11.11.1924, p. 3, col. 1.

54. - «Franz Werfel sendet uns folgende Zeilen», in Vorwärts, $n^{\circ} 572,4.12 .1924$, p. 2 (pas dans OU). 55. - «Die Stunde des Christus», Paulus unter den Juden als Maifestspiel, in: Prager Presse 8.5.1930, p. 8 (pas dans OU). Werfel reprend la même idée dans Historisches Drama und Gegenwart, OU p. 598.

56. - Dramen I, p. 401.

57. - Ibid., p. 455.

58. - Ibid., p. 414.

59. - Ibid.

60. - Axel Schalk, «F. Werf els Historie "Juarez und Maximilian". Schicksalsdrama, "Neue Sachlichkeit" oder die Formulierung eines paradoxen Geschichtsbild? in Wirkendes Wort, Bonn, $\mathrm{n}^{\circ} 1,1988$, p. 85.

61. - Norbert Abels, Franz Werfel, Hambourg, Rowohlts Bildmonographien, ${ }^{\circ}$ 472, 1990, p. 70.

62. - Th. Koebner, «Das Drama der Neuen Sachlichkeit und die Krise des Liberalismus», in : W. Rothe Hrsg. Die deutsche Literatur in der Weimarer Republik, Stuttgart, Reclam, 1974, p. 32-33.

63. - «Werfel se livre à des études d'une singulière ampleur. Ses connaissances historiques sont extraordinaires et il ne manque pas un iota à la parfaite reproduction des figurants de l'histoire mondiale qu'il a placés sur scène ». Commentaire de E.M. Salzer à la suite de l'interview de Werfel, «Wie mein "Reich Gottes in Böhmen" entstand», loc. cit. Sur l'exactitude des costumes v. H. Meister, op. cit., p. 130-131. Cela permit à Karl Kraus d'ironiser sur la scrupuleuse reproduction du couvre-chef de Maximilien! (Fackel. n 697-705, oct. 1925, p. 24).

64. - Verdi. Roman der Oper (1923), Francfort/M., S. Fischer, 1966, p. 221 (chap. 6).

65. - Walter Sokel, Der Literarische Expressionismus, Munich, Langen-Müller, 1970 (trad. de The Writer in extremis, Stanford, 1959), p. 167 et 271.

66. - OU, p. 229, 231 (Dramaturgie...), 591-592 \{Absage an den Expressionismus), 673 (Journal) et 702 (Autobiographische Skizze).

67. - Ibid., p. 229-231.

68. - Les six nouvelles dans : Erzählungen aus zwei Welten hrsg. von A. Klarmann, t. 2, Francfort/M., S. Fischer, 1952. Nouvelle édition : Die tanzenden Derwische. Erzählungen, Francfort/M., S. Fischer Taschenbuch Vlg., 1989, p. 103-211 et Die Entfremdung. Erzählungen, Fischer Taschenbuch Vlg., 1990. 
69. - W. Nagl.-J. Zeidler-E. Castle, Deutschösterreichische Literaturgeschichte, Vienne/Leipzig, Fromme Vlg., t. 4, 1937, p. 2249. Dès 1930, Der Tod des Kleinbürgers fut classé dans la Neue Sachlichkeit, cette nouvelle comptant parmi les œuvres sur « l'érosion monétaire » (Alois Bauer, «Vorläufiges zur Neuen Sachlichkeit», in Zeitschrift für Deutschkunde, vol. 44, 1930, p. 79).

70. - Erzählungen aus zwei Welten, t. 2, op. cit., p. 232 (Das Trauerhaus).

71. - Bertaux, op. cit., p. 311.

72. - R. Specht, «Der Abituriententag», in Neue Freie Presse, Vienne, 1.6.1928 (compte rendu).

73. - H. Schimmelpfeng Werfeis, «Abituriententag», in Die Christliche Welt, vol. 43, 1929, p. 131-133.

74. - Jost Hermand, «(๕dipus lost: Oder der im Massenleben der Zwanziger Jahre "aufgehobene"Vater-Sohn-Konflikt des Expressionismus», in R. Grimm-J. Hermand, op. cit., p. 220. 75. - Der Abituriententag, Francfort/M., Fischer Taschenbuch Vlg., 1990, p. 112-113 (chap. 5).

76. - Ibid., p. 141 (chap. 6).

77. - Ibid., p. 7 et 63 (chap. 3).

78. - Ibid., p. 23 (chap. 27).

79. - Ibid., p. 87 (chap. 47).

80. - «Was arbeiten Sie? Gespräch mit Franz Werfel». Interview par Willy Haas in Die Literarische Welt, Berlin, $\mathrm{n}^{\circ} 2,1926$, p. 33-35.

81. - Werfel, «Das Reich Gottes in Böhmen», interview dans Prager Presse 18.12.1930, p. 8 (puis Witiko 3, Cassel, 1931, p. 130-131).

82. - Barbara oder Die Frömmigkeit II, 2 (Francfort/M., S. Fischer, 1957, p. 1027).

83. - Ibid. II, 12 («Der Ruf nach Blut»).

84. - Franz Werfel, «Erklärung», in Der Vormarsch. Blätter der nationalistischen Jugend, Berlin, vol. 1, 1927-1928, p. 271.

85. - Gertrud Alexander, «Die neuere Dichtung», in Die Rote Fahne, vol. 4, 1921, n 264 (13.1). Reproduit dans W. Fähnders-M. Rector, Literatur im Klassenkampf, Zur proletarisch-revolutionären Literaturtheorie, Eine Dokumentation, Munich, Hanser, 1971, p. 90.

86. - Interview radiodiffusée de Piscator par H. Ihering in Die Literatur, vol. 1928-1929, p. 497-500, reproduit dans E. Piscator, Schriften, t. 2, Aufsätze, Reden, Gespräche, Berlin, Henschelverlag Kunst und Gesellschaft, 1968, p. 63.

87. - H. Ihering, Die getarnte Reaktion, loc. cit., dans Hans Mayer, op. cit., p. 656-657.

88. - O. Biha, «Der Tod des Spiessers. Dreimal Flucht aus der Wirklichkeit», in Die Rote Fahne, vol. $14, \mathrm{n}^{\circ} 177,13.9 .1931$ et «Die Ideologen des Kleinbürgertums», in Internationale Literatur, vol. 2, $\mathrm{n}^{\circ} 2$, 1932. R. Braune, «Herr Werfel zieht in den Krieg», in Die Linkskurve, vol. 4, $\mathrm{n}^{\circ} 2$, 1932. Ces trois articles cités dans Zur Tradition der sozialistischen Literatur in Deutschland. Eine Auswahl und Dokumentation, Berlin/Weimar, 1967, p. 777.

89. - G. Lukâcs, Grösse und Verfall des Expressionismus, in Internationale Literatur, $\mathrm{n}^{\circ} 1,1934$, p. 153-173. D'après G. Lukâcs, Werke, t. 4. Essays über Realismus. Probleme des Realismus 1, Neuwied/ Berlin, Luchterhand, 1971, p. 134-135.

90. - G. Lukâcs, Karl Marx und Friedrich Theodor Fischer, 1934, in Werke, op. cit., t. 10 : Probleme der Ästhetik, p. 301.

91. - Dramen I, p. 427.

92. - Ibid. II, p. 20.

93. - Ibid., p. 41.

94. - E. Bloch, Diskussionen über Expressionismus in Das Wort, vol. 6, 1938, p. 98-103.

95. - Julius Bab, «Bilanz des Dramas», in Die Volksbühne, vol. 5, 1930, n³, p. 100. Cité d'après Th. Koebner, loc. cit., p. 19.

96. - B. Brecht, Schriften zur Literatur und Kunst, Francfort/M., Suhrkamp, 1967, t. 1, 1920-1932, p. 70-72 et 250 .

97. - B. Brecht, op. cit., p. 44, 46-47. 
98. - Le poème de Küpper n'est pas reproduit dans les Schriften zur Literatur und Kunst de Brecht. On le trouvera dans Hans Mayer, op. cit., p. 446-447.

99. - Heinz Kindermann, «Vom Wesen der "Neuen Sachlichkeit"», in Jahrbuch des Freien Deutschen Hochstifts Frankfurt am Main, 1930, p. 354-386.

100. - OU, p. 16-40. De ce réalisme radical fait partie l'Américanisme, lequel est compté par Helmut Lethen parmi les composantes de la Neue Sachlichkeit. Chez Werfel, l'Américanisme représente d'une part une tentation des Années Vingt: les héros de Nicht der Mörder... et Der Abituriententag Karl et Adler émigrent finalement en Amérique ; les pièces Bocksgesang et Juarez und Maximilian présentent des personnages d'Américains à la fois cyniques et de bon sens. Mais pour le Verdi de Werfel, l'activisme américain («Die amerikanische Hätz») est un des aspects «lucifériens nordiques» (Verdi, op. cit., p. 194). A la même époque, Werfel compose la ballade Die Zeit der Riesen kommt, dans laquelle notre vieille culture est ravagée par d'affreux golems... parlant américain! (Das lyrische Werk, op. cit., p. 575-576). Sur quelques aspects de l'Amérique chez Werfel, v. Hans-Bernhard Moeller, «Amerika als Gegenbild bei Franz Werfel», in Literatur und Kritik, n 81, 1974, p. 42-48. Sur le problème de l'américanisme des Années Vingt en général, v. l'article fondamental de Gérard Imhoff, «Der Amerikanismus der zwanziger Jahre oder die Versuchung eines alternativen Weltildes», in Revue d'Allemagne, t. XXII, n 3, 1990 (juilletseptembre), p. 427-437.

101. - OU, p. 348 .

102. - Ibid., p. 18.

103. - Sur Zola : OU, p. 18, 38, 345, 441, 664, 665, 678, 684, 687. Essai sur Zola : «Emile Zola », dans: Wilhelm Herzog, Menschen, denen ich begegnete, Berne/Munich, Francke Vlg., 1959, p. 445-447. Paru en français dans Présence de Zola, Paris, Fasquelle, 1952.

104. - OU, p. 478.

105. - Dramen I, p. 424 et II, p. 64.

106. - Werfel sur le Romantisme: OU, p. 329, 401, 753-755 ; Verdi, op. cit., p. 27-28, 87, 196 ; Ein Bildnis Giuseppe Verdis, in : Giuseppe Verdi, Briefe, hrsg. und eingeh von F. Werfel, übers, von Paul Stefan, Vienne, Paul Zsolnay, 1926, p.14-15; Verdi und die Romantik, in: Die Literarische Welt, Berlin, 20.10.1926, p. 4.

107. - Lévy sur le Romantisme : Le Testament de Dieu, Paris, Grasset, 1977, p. 10, 25, 77, 122-123, $145,161$.

108. - Ibid., p. 49.

109. - OU, p. 210 et 214.

110. - Ibid., p. 191.

111. - Bertaux, op. cit., p. 315-316.

112. - Ibid.

\section{RÉSUMÉS}

Chez Franz Werfel, la production des Années Vingt est dominée par les drames Spiegelmensch, Bocksgesang, Schweiger, Juarez und Maximilian, Paulus unter den Juden et Das Reich Gottes in Böhmen. Leur structure commune oppose des révolutionnaires réalistes à des révolutionnaires extrémistes. Ils représentent une phase de réflexion politique et donc un dépassement de l'extase expressionniste. Les nouvelles de 1927-1928, dont Der Abituriententag, ainsi que le roman Barbara 
oder Die Frömmigkeit (1929) s'inscrivent dans la même ligne par leur message de lucidité et de non violence. La modération de Werfel lui a attiré l'hostilité de la critique marxiste. Le désaveu qu'il formule contre le radicalisme annonce le théâtre «existentialiste" français et la Nouvelle Philosophie. La période de Neue Sachlichkeit prépare la dernière phase de production de Werfel, d'inspiration authentiquement religieuse en même temps que respectueuse des réalités.

In Werf eis Schaffen der Zwanziger Jahre überragen die Dramen Spiegelmensch, Bocksgesang, Schweiger, Juarez und Maximilian, Paulus unter den Juden und Das Reich Gottes in Böhmen. Alle leben von der Opposition zwischen radikalen und nüchternen Revolutionären. Sie bilden eine Phase der politischen Besinnung und somit eine Ueberwindung der expressionistischen Ueberspanntheit. Die Novellen von 1927-1928, darunter Der Abituriententag, so wie der Roman Barbara oder Die Frömmigkeit (1929) weisen in dieselbe Richtung durch ihre Moral der Erkenntnis und der Gewaltlosigkeit. Werf eis Mässigung forderte Ablehnung von seiten der marxistischen Kritik heraus. Die Absage an den Radikalismus nimmt das Theater der französischen Existentialisten und die Position der sog. Nouvelle Philosophie der siebziger Jahre vorweg. Die Neue Sachlichkeit bei Werfel ist die Vorbereitung auf seine lelzte, sowohl religiöse als auch realitätsnahe Schaffensperiode.

\section{AUTEUR}

\section{MICHEL REFFET}

Université de Dijon 